\title{
Evaluación de las habilidades requeridas en el análisis de un sistema organizacional
}

\section{Evaluation of the skills required in the analysis of an organizational system}

Blanca Carballo Mendívil (bcarballom@gmail.com)

Profesor por asignatura Instituto Tecnológico de Sonora (México)

\author{
Alejandro Arellano González (alejandro.arellano@itson.edu.mx) \\ Profesor investigador en Instituto Tecnológico de Sonora (México) \\ José Mario Salomón González (josems16@gmail.com) \\ Exalumno tesista en Instituto Tecnológico de Sonora (México) \\ http://dx.doi.org/10.12795/EDUCADE.2014.105.06
}

\begin{abstract}
RESUMEN: Se determina si alumnos del Instituto Tecnológico de Sonora, México, desarrollan las competencias para realizar un análisis de procesos en una organización. Se aplicó un instrumento tipo rúbrica de 14 ítems con escala Likert de cuatro niveles, para evaluar 98 proyectos desarrollados por los alumnos, encontrando que cuando cursan la materia de análisis de sistemas demuestran capacidad para describir una empresa y analizar sus procesos, habilidades básicas en su formación académica, y que deberían seguir exhibiendo más adelante en su proceso formativo, pero no lo hacen, es decir, aunque sepan qué es lo que tienen que hacer y cómo hacerlo no lo realizan, ya sea porque no sabe cómo o porque no se les solicita. Se debe fomentar el uso de las competencias que ya han sido desarrolladas para fortalecerlas y se cumpla con las expectativas que se tienen de egresado.
\end{abstract}

PALABRAS CLAVE: Evaluación basada en el desempeño, Educación basada en competencias, Habilidad académica, Aprendizaje activo.

\begin{abstract}
It is determined whether students from the Technological Institute of Sonora, Mexico, develop the skills to perform an analysis of processes in an organization. An instrument with 14 items in Likert scale of four levels was applied to assess 98 projects developed by students. The findings are that when they take the course of systems analysis they demonstrate the ability to describe and analyse business processes, basic skills in their academic training, and should continue to show later in the educational process, but they do not, that is, even if they know what to do and how to do it, do not, either because they do not know how or because it is not requested. It should encourage the use of skills that have been developed to strengthen and fulfil the expectations we have of a graduate.
\end{abstract}

KEYWORDS: Performance Based Assessment, Competency Based Education, Academic Ability, Active Learning. 


\section{INTRODUCCIÓN}

Se tienen muchas expectativas de las universidades en su rol de ser conciencia de la comunidad donde se encuentra, no sólo para cumplir con su cometido generalmente establecido en su misión: el desempeño de sus funciones sustantivas para generar los profesionistas que serán motores de desarrollo en las organizaciones en que se inserten, sino que además estas instituciones debe considerar, en el diseño de sus planes y programas de estudio, los fenómenos que afectan a la colectividad para incidir positivamente en su solución y cierre de brechas en la realidad social, y así gradualmente mejorar la calidad de vida a sus habitantes (Méndez-Fregozo, 2005:418).

Así pues, las universidades están sujetas a una serie de presiones para que cumplan su función social, que implica no solamente formar profesionistas altamente calificados, generar tecnología y conocimientos de frontera así como extender sus programas culturales y deportivos a la comunidad, además deben mostrar evidencias respecto a la pertinencia de sus resultados y eficiencia en el manejo de sus recursos.

Por ello, el Instituto Tecnológico de Sonora (ITSON) de México, consciente de su responsabilidad con la comunidad, desde el año 2002 orientó su programas educativos hacía el desarrollo de competencias profesionales, que luego reestructuró en 2009, lo que ha implicado el reto de buscar los mecanismos para generar situaciones de aprendizaje significativo para sus estudiantes, explorando desde entonces diversas estrategias de aprendizaje que aseguren que su egresado se caracterice por el dominio de las competencias correspondientes a su profesión (Instituto Tecnológico de Sonora, 2009:1).

En la literatura especializada se han planteado diferentes modelos y estrategias para lograr este desarrollo de competencias, sin embargo cada universidad tiene sus propias características y su comunidad diferente naturaleza, por lo que se requiere adaptar las diferentes propuestas empíricas y modelos teóricos para generar el más adecuado acorde a los objetivos de cada programa educativo que le permita cumplir con sus indicadores críticos, destacando la pertinencia y calidad de sus egresados.

Sin embargo, independientemente de la universidad o del programa educativo que curse, si se espera que el egresado sea competente en el estudio de organizaciones, se considera que este domine la competencia básica del pensamiento sistémico, que le permita al alumno realizar el análisis y diseño del sistema organizacional que aborde, a través de un trabajo colaborativo desarrollado en una empresa. En la literatura se documentan diversas experiencias que ponen de manifiesto la enorme relevancia de la capacidad de trabajo en equipo para el logro de los resultados esperados, no sólo durante el proceso formativo donde se promueve la adquisición de estas competencias necesarias para desarrollar tareas en un entorno empresarial, sino en el ámbito profesional donde dichas competencias son puestas en práctica en una realidad sin supervisión académica.

Entre estas experiencias se puede comentar el caso del estudio desarrollado por García-Fernández y Cordero-Borjas (2007:17,30-31) que demuestra que los equipos de trabajo son formas organizativas propias de la "nueva era", donde se espera que las personas interactúen para generar, transferir y utilizar el conocimiento en función de obtener los rendimientos esperados de las inversiones realizadas en las organizaciones. Asimismo estos autores indican que si las organizaciones no implementan cambios económico-organizativos para adecuar el funcionamiento de la empresa a las nuevas condiciones de trabajo, en la mayoría de los casos no obtienen la productividad esperada y por consiguiente, los rendimientos de sus inversiones. 
Asimismo, Alarcón-Ortiz y Freire-Cruz $(2012: 18,28)$ explican que el desempeño de las personas dentro de las organizaciones está determinado por ciertos factores que mantienen unido a un grupo organizacional, que se consideran indicadores culturales: solidaridad y sociabilidad, que se manifiestan al abordar situaciones problemáticas típicas del trabajo en equipo en cualquier organización. De igual manera, los autores presentan una propuesta para resolver conflictos del trabajo en equipo, tales como: estimular a los integrantes del equipo a escucharse mutuamente y considerar diferentes puntos de vista, cuestionar objetivamente los supuestos de los demás, expresar temas controversiales sin temor a represalias, expresar sus preocupaciones describiendo la situación con el mayor detalle posible, mantener la discusión en plano impersonal, no a hacer supuestos respecto a la motivación de comportamiento no satisfactorios, dar sugerencias concretas para mejorar, entre otras.

De igual manera, Ardila-Soto \& Gómez-Chiñas $(2005: 147,162)$ mencionan que el trabajo en equipo bien aplicado constituye una excelente herramienta estratégica para la dirección de empresas que permite lograr sus objetivos a través de la integración y motivación de los miembros del equipo; sin embargo hay empresas que han fracasado con su aplicación, por lo que realizaron un estudio para comparar el concepto teórico del trabajo en equipo con el concepto empírico que tienen los directivos de empresas colombianas, con objeto de determinar si las empresas aplican realmente el trabajo en equipo o simplemente creen estar haciéndolo de acuerdo a su conveniencia. Los resultados muestran que aunque la mayoría de los directivos entrevistados aseguró que en su organización se trabaja en equipo, no existe un concepto claro de trabajo en equipo, aunque un alto porcentaje de los empresarios piensan que están aplicando innovación administrativa en lo referente a trabajo en equipo, posiblemente no lo estén haciendo.

Por su parte, Pech-Pech, Castillo-León y Echeverría-Echeverría (2005:12) realizaron un diagnóstico para identificar el estado actual de las competencias profesionales del personal para el trabajo en equipos interdisciplinarios en una organización del sector salud, encontrando que existe la necesidad de desarrollar conocimientos e incrementar habilidades y actitudes sobre aspectos como interdisciplina, toma de decisiones, liderazgo, manejo de conflictos y comunicación para optimizar el trabajo en equipos interdisciplinarios.

En este sentido, y dada la importancia del trabajo colaborativo, en todo programa educativo del ITSON donde se aborde una organización de manera colaborativa, se considera necesario el desarrollo de la competencia genérica del pensamiento de sistemas, como ya se ha comentado, por lo que se ha instrumentado a través de una estrategia de Aprendizaje Basada en Problemas (ABP) que ha planteado el reto de que el alumno seleccione e intervenga una pequeña empresa como objeto de estudio (Arellano-González \& Carballo-Mendívil, 2011):45). Sin embargo, este enfoque no ha sido implementado en el resto de los bloques de materias "cliente" (donde se hace análisis y diseños específicos de la disciplina), ni se le ha dado seguimiento al desempeño del alumno para poder establecer propuestas de mejora.

Es de relevancia mencionar también que en el programa educativo donde se ha instrumentado esta estrategia, actualmente se presentan una serie de situaciones no deseadas, tal como el rezago educativo en sus estudiantes y el bajo desempeño de los mismos en estas materias básicas del programa, y aunque Arellano-González, RíosVázquez, Carballo-Mendívil, Montero-Quintero y Tapia-Mimila (2013):31) presentan información diagnóstica respecto al problema del rezago (aproximadamente solamente el $20 \%$ de la primer cohorte generacional egresó oportunamente) y los índices de reprobación $(81 \%$ en el primer curso del bloque de sistemas donde se desarrolla la competencia genérica previamente descrita), aún no se ha medido el nivel de competencia que han desarrollado los alumnos durante su progreso por el 
programa, y por lo tanto no se tiene información adecuada para la toma de decisiones que promueven la mejora de sus planes y programas y con esto asegurarse de egresar a profesionistas que la sociedad demanda.

Así pues, el estado en el que se encuentra el alumnado de esta universidad es preocupante, pues se puede notar una gran brecha entre su desarrollo actual y el ideal, uno de los principales indicadores de esta situación es un notable índice de reprobación y rezago, particularmente en el bloque de sistemas y específicamente en dos cursos: análisis de sistemas (AS) y diseño de sistemas (DS). Una situación que no debería prevalecer en los siguientes semestres si se busca mejorar la competitividad de los egresados de ITSON.

Entre los maestros responsables de las academias de sistemas ha tomado más fuerza la preocupación respecto a que los alumnos no están desarrollando dichas competencias. Para poder respaldar esta preocupación es necesario evaluar de manera consistente el nivel de competencia que están desarrollando los alumnos, respecto a lo esperado por los cursos pertenecientes al bloque de sistemas, para generar información que permita tomar las medidas necesarias que promuevan la mejora del programa educativo. Por lo anterior, se detona la siguiente pregunta de investigación: ¿̇Cómo determinar el nivel de habilidades adquirida por los alumnos respecto al análisis de un sistema organizacional? Y para responder a esta pregunta se estableció como objetivo, diseñar un mecanismo de evaluación respecto a las habilidades requeridas en el análisis de un sistema organizacional, para determinar de manera formal y permanente el nivel de desempeño alcanzado respecto a una competencia básica adquirida por los alumnos.

Con el proyecto que aquí se reporta se buscó diseñar un mecanismo de evaluación que permitiera determinar en nivel de desempeño de los alumnos cuando se aborda un sistema organizacional: el análisis de un sistema, el cual es un elemento de una competencia básica que se debe desarrollar en cualquier programa donde se estudien organizaciones. Asimismo, con la información que se obtenga de la aplicación de este mecanismo de evaluación se podrá conocer, de manera eficiente y con las evidencias necesarias, la realidad que se vive en el programa educativo alrededor de esta habilidad básica de análisis que deben desarrollar los alumnos, que aunque se ha observado que existe un bajo desempeño por parte del estudiante, no se ha sistematizado una forma de evaluación que pueda ser aplicada de manera permanente en distintos momentos, de tal manera que se pueda dar seguimiento a los resultados que logren obtener a partir de las acciones de mejora que se tomen al interior de las academias.

Si no se hubiera llevado a cabo este proyecto se seguiría sin tener información que fundamente la toma de decisiones respecto a la administración del programa, el diseño de los cursos y la impartición de los mismos, lo cual supone que se seguirá manteniendo el problema presentado en la actualidad respecto al bajo desempeño académico de los alumnos, alto índices de reprobación y rezago educativo.

\section{FUNDAMENTACIÓN TEÓRICA}

\subsection{Desarrollo de competencias profesionales}

Hablar de competencias no es nada nuevo. De hecho, desde la etapa medieval se usa el concepto de competencias para señalar la adquisición de habilidades por parte de los aprendices de diversos oficios en su trabajo con el patrón (LópezHernández \& Abelló-Planas, 2007):34). Las competencias son capacidades que el individuo desarrolla, para un trabajo, una profesión, estas pueden lograrse adquiridas 
por formación, práctica, estudio, ejercicio, posesión de conocimientos, destrezas, habilidades o actitudes que permitan dominio en la realización de una actividad.

Una de las definiciones más simples pero claras que se pueden encontrar sobre competencias es la establecida por López-Hernández y Abelló-Planas (2007):35) quienes dicen que en conjunto, se trata de un manojo de atributos, de una etiqueta para una colección de conocimientos, habilidades, actitudes, tareas y resultados.

Las habilidades por su parte, se trata de una capacidad adquirida para realizar una tarea en particular con el fin de alcanzar un objetivo preciso. Es el resultado de un aprendizaje a menudo largo que depende del conjunto de recursos de que se dispone el individuo, es decir, su capacidad para transformarse y su repertorio de conductas (Durand en (Hernández-Alvarez, y otros, 2004):139).

\subsubsection{Implementación del modelo por competencias en el Instituto Tecnológico de Sonora}

Desde el año 2002 el ITSON implementa en sus programas educativos un modelo curricular basado en competencias, detectando en la práctica que este modelo exige centrarse en el aprendizaje para el logro de competencias profesionales a través de la interacción alumno-maestro-comunidad. Luego, en el 2009 los programas se rediseñan considerando un modelo centrado en aprender a utilizar el conocimiento en diversas situaciones de aprendizaje y con vínculo directo con la comunidad, por lo cual estos programas se enfrentan al reto de promover esta interacción para que el estudiante no sólo obtenga conocimientos, sino que desarrolle habilidades, actitudes y valores requeridos para un buen desempeño laboral (Instituto Tecnológico de Sonora, 2009):1).

Esta orientación de los programas educativos hacía el desarrollo de competencias profesionales, ha implicado el reto de buscar los mecanismos para generar situaciones de aprendizaje significativo para sus estudiantes, explorando desde entonces diversas estrategias de aprendizaje que aseguren que su egresado se caracterice por el dominio de las competencias correspondientes a su profesión, tal es el caso del desarrollo de iniciativas estratégicas cuyo objetivo ha sido generar un espacio real donde los alumnos puedan desarrollar las competencias que se han definido para su programa educativo (Guerra-López \& Rodríguez-Villanueva, 2005):56).

Particularmente, en uno de los programas educativos de ITSON orientado al estudio de organizaciones, después de la reestructuración curricular llevada a cabo en el 2009 replanteó su plan de estudios para establecer el compromiso de formar egresados que no sólo dominen un conjunto de competencias profesionales relacionados con la disciplina, sino además se espera que desarrollen una competencia que se considera básica para cualquier profesionista que estudie a las organizaciones, llamada pensamiento sistémico, a través de la cual se pretende que el alumno sea capaz de realizar el análisis de procesos y el diseño de soluciones bajo un enfoque de sistemas.

Se considera que para desarrollar cualquiera de estas competencias únicamente se puede lograr a través del estudio de organizaciones reales a través de proyectos, lo cual es reportado en la literatura como una estrategia con múltiples ventajas, ya que elaborar un proyecto permite y alienta a los estudiantes a experimentar, aprender de sus errores y enfrentar retos difíciles e inesperados; estimulando el desarrollo de habilidades para resolver situaciones reales, con lo cual se motivan a aprender; además, promueve que aprendan a aprender el uno del otro y a ayudar a que sus compañeros aprendan, evaluar el trabajo y dar retroalimentación constructiva (Galeana de la O., 2006; Maldonado-Pérez, 2008). 


\subsubsection{El Aprendizaje Basada en Proyectos (ABP) y su contribución en la formación de egresados competentes}

El Aprendizaje Basado en Proyectos (ABP) es una estrategia educativa que promueve el aprendizaje colaborativo en entornos reales. Esta estrategia busca que los estudiantes desarrollen un trabajo en equipo en el que se debe aplicar el conocimiento teórico para realizar algo práctico. Cuando el objeto de estudio es una empresa, normalmente debe proponer y/o implementar algún cambio en ella.

En la literatura se reportan diversas experiencias sobre esta estrategia que concluyen que permite generar experiencias positivas, aunque es posible que los estudiantes aprendan menos conocimientos, pero sí desarrollan otras habilidades como trabajo en equipo, búsqueda de información, uso de herramientas colaborativas, gestión del tiempo, etc. (Maldonado-Pérez, 2008; Labra-Gayo y otros, 2006). Esta estrategia puede aplicarse en asignaturas con sesiones prácticas o de laboratorio, así como en sesiones de teoría (García-Almiñana y Amante-García, 2006), pero hace una mala combinación con una evaluación fuertemente basada en exámenes (Valero-García, 2005), por lo que es más fácil implementarlo en carreras nuevas, en vez de transformar carreras tradicionales que no acostumbran a desarrollar el ABP (Kolmos, 2004).

Como ya se ha mencionado, la academia de Sistemas del ITSON, instancia que administra el bloque de materias orientadas al desarrollo de la competencia básica del pensamiento de sistemas, ha instrumentado una estrategia de Aprendizaje Basada en Problemas (ABP), que ha planteado el reto de que el alumno seleccione e intervenga una micro o pequeña empresa como objeto de estudio, de tal manera que desarrolle una competencia básica en su desempeño profesional. Sin embargo, este tipo de estrategias tiene una serie de implicaciones positivas y negativas en cada etapa del desarrollo de los cursos, destacándose que si bien el enfoque ABP ha demostrado ser una estrategia efectiva para lograr en el alumno el desarrollo de competencias, tal como lo reporta la literatura consultada, no es sencillo de implementar, sobre todo sin los medios adecuados y una estrategia bien establecida que indique qué hacer cuando los proyectos se complican y los alumnos requieran orientación para cumplir las metas establecidas (Arellano-González \& CarballoMendívil, 2011):50).

En primer lugar, una premisa para que el $A B P$ sea efectivo, es que debe haber un programa de cursos diseñado acorde al enfoque metodológico que permita desde un inicio del semestre trabajar con el proyecto y que el desarrollo ocurra de manera continua hasta que este concluya, solo es necesario tener claro la competencia a desarrollar en el alumno y en el desglose funcional no perder de vista el enfoque al bajarlo hasta el nivel de elementos de competencia. Además, el enfoque ABP requiere que el profesor se someta a un reto permanente con los proyectos desarrollados por los alumnos ya que no sabe con qué dudas llegarán, y por lo mismo no solo debe manejar los temas teóricos con propiedad, también debe tener una formación metodológica adecuada y sobre todo, manejar el método de la dialéctica, ya que debe aceptar abiertamente que no tiene todas las respuestas a las dudas pero si puede orientar con preguntas que permitan buscar respuestas y soluciones ingeniosas.

Se considera que estas premisas y algunos de los retos han sido superados por la academia de sistemas que participa en el programa, ya que tres de los cuatro cursos que lo integran han sido diseñados bajo un enfoque de proyectos, se han generado materiales que apoyan metodológicamente en el cumplimiento de la competencia, y se ha habilitado a todos los profesores para que puedan orientar a los alumnos en el proceso. 
Lo anterior también lo secunda la experiencia que ha generado Alfaro-Tanco, Rodríguez-Chacón y Amorrortu-Gervasio (2014:1) en la Universidad de Navarra en España, con el curso de Dirección de Operaciones, quienes además de buscar lograr que el alumno adquiera los conocimientos básicos en este tema, se busca a la vez que desarrolle competencias necesarias para su incorporación en el mercado laboral y establezca un contacto real con la empresa. Estos autores indica que los modelos de aprendizaje basados en proyectos tienen su base científica en el proceso de generación de conocimiento, en el cual los estudiantes no son receptores pasivos de conocimiento, sino que construyen conocimiento sobre la base de lo que ellos realmente saben y han experimentado, lo que se consigue a través de la participación e interacción con otros.

Tal como lo ha generado la academia de sistemas del ITSON, que ha trabajado durante cuatro años con el ABP, estos autores muestran los resultados obtenidos en los últimos seis años y concluyen que el plantearle al alumno que resuelva un problema en una empresa real de una manera semi-autónoma hace que éste se plantee cuestiones que con las clases magistrales no lo haría. Además, debido al seguimiento realizado a través de los hitos por el profesor, los equipos son capaces de corregir sus errores y el conocimiento de la materia se refuerza.

\subsection{Evaluación educativa}

Según Tenbrink (2006):17) evaluar es asignar un valor a algo y en educación, normalmente esto significa juzgar a un estudiante, profesor o programa educativo. Por ejemplo, los profesores emiten muchos juicios en el proceso de evaluar los logros de sus alumnos y una forma común del juicio es la calificación, haciendo con esto públicos sus juicios sobre las realizaciones académicas de sus alumnos.

La evaluación es un proceso y a la vez un producto, cuya aplicación permite estimar el grado en el que un proceso educativo favorece el logro de las metas para los que fue creado, tales como las competencias que pretende lograr un currículo basado en este modelo.

La evaluación tiene como gran finalidad educativa el seguimiento del proceso de enseñanza y aprendizaje, para mejorarlo y poder reconducirlo y adecuarlo a las necesidades del alumnado, con el fin regulador del proceso de enseñanza y aprendizaje, y no como un instrumento de selección del alumnado por sus rendimientos.

\subsubsection{La rúbrica como instrumento para la evaluación}

En el contexto evaluativo una rúbrica es una tabla que contiene los parámetros de evaluación y permite aunar criterios de evaluación, niveles de logro y descriptores (Capote \& Sosa, 2006):16), la cual facilita el proceso de calificación del desempeño de un estudiante con el listado de criterios permite valorar el aprendizaje, los conocimientos y/o las competencias, logrados en un trabajo o asignatura.

Con este fin establece una gradación (niveles) de la calidad de los diferentes criterios con los que se puede desarrollar un objetivo, una competencia, un contenido o cualquier otro tipo de tarea que se lleve a cabo en el proceso de aprendizaje.

\subsubsection{Confiabilidad de instrumentos}

Como todo instrumento, una rúbrica debe someterse a pruebas de confiabilidad para determinar el grado con el cual el instrumento prueba su consistencia, por los resultados que produce al aplicarlo repetidamente al objeto de estudio. La confiabilidad es la capacidad del mismo instrumento para producir resultados congruentes cuando se aplica por segunda vez, en condiciones tan parecidas como 
sea posible. Es decir, el instrumento arroja medidas congruentes de una medición a la siguiente (Bernal-Torres, 2006):214).

Generalmente esta confiablidad se determina con el estadístico alfa de Cronbach que puede considerarse como un coeficiente de correlación. Una interpretación de sus resultados indicaría que si los diferentes ítems de una escala están midiendo una realidad común, las respuestas a estos ítems tendrían que presentar una elevada correlación entre sí: en caso contrario, la existencia de una baja correlación entre algunos ítems mostraría que algunas declaraciones de la escala no son medidas fiables del constructo (Molina-Morales, Martínez-Fernández, Ares-Vázquez, \& EmilHoffmann, 2008):73).

El valor de alfa de Cronbach puede oscilar entre 0 y 1 ; si es 0 significa que las puntuaciones de los ítems individuales no están correlacionadas con las de todos los demás. Por el contrario, ente más alto el valor del alfa significa una mayor correlación entre los distintos ítems, aumentando así la fiabilidad de la escala. Sin embargo, no existe un consenso entre los autores sobre cuál debe ser el valor a partir del cual se puede considerar que una escala presenta una fiabilidad aceptable. Por ejemplo, Nunnally en Molina-Morales, Martínez-Fernández, Ares-Vázquez y Emil-Hoffmann (2008):73) señala que, en investigaciones de naturaleza exploratoria, el valor mínimo recomendado se sitúa en 0.70 y en estudios no exploratorios el valor recomendado es 0.80. Otros autores no distinguen entre tipos de estudios, considerando que para la mayoría de las investigaciones, un valor igual o inferior a 0.60 indica una fiabilidad interna no satisfactoria. El valor dependerá en cierta medida de la cantidad de elementos de la prueba y el número de participantes, pero 0.75 es un valor de compromiso razonable para tomar como punto de referencia (Molina-Morales, Martínez-Fernández, Ares-Vázquez, \& Emil-Hoffmann, 2008):73).

El cálculo del alfa de Cronbach se basa en el número de elementos (es decir, el número de preguntas en un cuestionario) y la media de correlación inter-ítem. Si se asume que las preguntas están midiendo una verdadera calificación (por ejemplo el verdadero nivel de felicidad de una persona) la cuestión cada individuo medirá la verdadera calificación más una cierta cantidad de error aleatorio. Una alta correlación entre los diferentes elementos que indiquen que se está midiendo lo mismo que sólo habrá pequeños valores para el error. Una baja correlación indica que hay una gran cantidad de error y los elementos no son fiables para la medición de la misma cosa.

\subsubsection{La validez de los instrumentos}

Mientras que la confiabilidad, relacionada con la consistencia interna y la precisión de las medidas que se obtienen con el instrumento, se refiere a la confianza que se concede a los datos, la validez se refiere al grado en que el instrumento mide lo que se pretende medir, según lo indicado por Moreira y Lang Silveira citado en Lucero y Meza (2002):1).

La validez puede examinarse desde diferentes perspectivas: validez general, validez de contenido y validez de constructo. La validez general se relaciona con el juicio que se hace respecto del grado en que el instrumento de medición mide lo que debe medir. Este juicio consiste en tener una idea clara de la variable que desea medirse y evaluar si las preguntas o ítems del instrumento en realidad la miden; por su parte, la validez de contenido se refiere al juicio sobre el grado en que el instrumento representa la variable objeto de medición, es decir, el grado en que representa el universo de la variable objeto de estudio; por último, en la validez relacionada con el constructo, el instrumento se juzga respecto del grado en que una medición se relaciona consistentemente con otras mediciones sobre conceptos que están midiéndose. 
El índice de Kaiser-Meyer-Oklin es una medida de adecuación de muestra que permite comparar las magnitudes de los coeficientes de correlación observados con las magnitudes de correlación parcial. De acuerdo con Kaiser citado por Apellániz y Amayra (2008):59), el baremo de evaluación del índice KMO sería el siguiente: 1 a 0.90 es Muy bueno; de 0.90 a 0.80 es meritorio; de 0.80 a 0.70 es Mediano; de 0.70 a 0.60 es mediocre; de 0.60 a 0.50 es bajo y menor que 0.50 es inaceptable. De acuerdo a Apellániz y Amayra (2008):59), se considera que la existencia de valores bajos en el índice KMO desaconseja la aplicación del análisis factorial.

El análisis factorial supone que hay una parte común de la variabilidad de las variables, explicada por factores comunes no observables. Cada variable tiene una parte de su variabilidad no común propia de cada variable; a esta variabilidad no común será referida como factor único. Se asume que los factores únicos correspondientes a las variables son independientes entre sí (Alvarez-Cáceres, 1995):240).

Al analizar factorialmente un conjunto de datos el investigador podría carecer de hipótesis específicas respecto a los parámetros del modelo, y buscar simplemente una estructura común subyacente en los datos. El uso del análisis factorial sería exploratorio, pero si el investigador parte de la hipótesis de que existe un número determinado de factores, los cuales tienen un significado determinado, el análisis factorial sería confirmatorio.

\section{METODOLOGÍA}

\subsection{Sujetos bajo estudio}

Los objetos de estudio en este proyecto fueron 97 evidencias disponibles de tipo producto que presentaron alumnos de un programa educativo de ITSON orientado al estudio de organizaciones, donde se documenta la experiencia en el desarrollo de un proyecto, que incluye un componente de análisis de un sistema organizacional, generadas por ellos desde agosto 2010 hasta mayo 2013, en dos tipos de cursos: 59 proyectos del curso de Análisis de Sistemas (curso 1), 17 proyectos del curso de Gestión de la demanda e inventarios (curso 2) y el resto de otros cursos del bloque de logística tales como planeación de sistemas logísticos y administración de sistemas de almacenamiento (21 proyectos).

\subsection{Instrumento de medición}

Se diseñó un instrumento de evaluación tipo rúbrica, cuya estructura puede verse en la Tabla 1, el cual permite valorar 14 elementos que debería incluirse en cualquier análisis de un sistema organizacional según los expertos en materia de análisis, los cuales son ponderados según su importancia, y donde se establecía una escala de Likert que denotaba la gradación de cuatro niveles:

- Excelente: es la mayor valoración con un puntaje de 4 puntos, el producto contiene todos los requisitos que pide la rúbrica de manera impecable.

- Regular: valoración intermedia alta con un puntaje de 3 puntos, el producto cumple con los requisitos pero alguno no está desarrollado correctamente.

- Por debajo de las especificaciones: valoración intermedia baja con un puntaje de 2 puntos, el producto carece de varios requisitos.

- No desarrollado: es la menor valoración con un puntaje de 1 punto, el producto no fue desarrollado o los errores son tales que necesita volverse a hacer. 
De acuerdo con el análisis estadístico realizado utilizando el paquete de software estadístico SPSS Statistics 20 a los datos obtenidos con la aplicación de este instrumento, el alfa de Cronbach es del $83.9 \%$ lo cual indica una buena confiabilidad del instrumento y el índice de Kaiser-Meyer-Olkin (KMO) es de 0.757 lo que indica una validez en el rango de "muy bueno", de acuerdo a Apellániz y Amayra (2008):59).

TABLA 1 ESTRUCTURA DEL INSTRUMENTO DE MEDICIÓN TIPO RÚBRICA.

\begin{tabular}{lll}
\hline Competencia & Elemento & Calificación* \\
\hline Describir un sistema & 1 & Tamaño de empresa \\
organizacional & 2 & Sector y subsector al que pertenece \\
& 3 & Organigrama \\
& 4 & Diagrama de flujo \\
& 5 & Localización \\
& 6 & Lay-out \\
& 7 & Evolución \\
& 8 & Productos/Clientes \\
& 9 & Recursos/Proveedores \\
& 10 & Contexto Organizacional: PESTE \\
Analizar un sistema & 11 & Mapeo organizacional \\
organizacional & 12 & Procesos organizacionales idealizados \\
& 13 & Sintomatología identificada en la organización \\
& 14 & Formulación del problema \\
\hline
\end{tabular}

*Escala de medición: Excelente=4 puntos; Regular=3 puntos; Por debajo de las especificaciones=2 puntos; No desarrollado= 1 punto

Fuente: Diseñado por los investigadores.

En las tablas 2 y 3 se presentan los grupos de ítems formados por el software estadístico. En la tabla 2 se muestran los valores mayores obtenidos del KMO en cada ítem procesado, con esta información es posible agrupar dichos ítems, lo cual se presenta en la tabla 3.

TABLA 2 MATRIZ DE COMPONENTES ROTADA.

\begin{tabular}{|c|c|c|c|c|}
\hline & \multicolumn{4}{|c|}{ Componente } \\
\hline & 1 & 2 & 3 & 4 \\
\hline Tamaño de empresa identificado & & & .748 & \\
\hline Sector y subsector al que pertenece & & & .805 & \\
\hline Organigrama de la empresa bajo estudio & & & & .460 \\
\hline Diagrama de flujo & & & & .698 \\
\hline Localización de la empresa & & & & .494 \\
\hline Lay-out & & & & .798 \\
\hline Evolución de la empresa & & & .493 & \\
\hline Productos/Clientes & & .519 & & \\
\hline Recursos/Proveedores & & .446 & & \\
\hline Contexto Organizacional: PESTE & & .824 & & \\
\hline Mapeo organizacional & .587 & & & \\
\hline Procesos organizacionales idealizados & & .808 & & \\
\hline Sintomatología identificada en la organización & .783 & & & \\
\hline Formulación del problema & .814 & & & \\
\hline
\end{tabular}

Fuente: Elaborado a partir de datos recolectados por los investigadores. 
Como se puede observar en la tabla 3 se han formado cuatro grupos de ítems, los cuales de acuerdo al análisis realizado se afectan entre sí, esta información puede resultar de mucha utilidad a la hora de hacer modificaciones en la metodología que se utiliza para orientar el desarrollo de los proyectos que elaboran los alumnos.

TABLA 3 Agrupación de ÍTEMS.

\begin{tabular}{|c|c|c|c|}
\hline 1 & 2 & 3 & 4 \\
\hline Mapeo organizacional & Productos / Clientes & $\begin{array}{l}\text { Tamaño de empresa } \\
\text { identificado }\end{array}$ & $\begin{array}{l}\text { Organigrama de la } \\
\text { empresa bajo } \\
\text { estudio }\end{array}$ \\
\hline $\begin{array}{l}\text { Sintomatología } \\
\text { identificada } \\
\text { en la organización }\end{array}$ & $\begin{array}{l}\text { Recursos / } \\
\text { Proveedores }\end{array}$ & $\begin{array}{l}\text { Sector y subsector al } \\
\text { que pertenece }\end{array}$ & Diagrama de flujo \\
\hline \multirow[t]{2}{*}{$\begin{array}{l}\text { Formulación del } \\
\text { problema }\end{array}$} & Contexto: PESTE & $\begin{array}{l}\text { Evolución de la } \\
\text { empresa }\end{array}$ & $\begin{array}{l}\text { Localización de la } \\
\text { empresa }\end{array}$ \\
\hline & $\begin{array}{l}\text { Procesos } \\
\text { organizacionales } \\
\text { idealizados }\end{array}$ & & Lay-out \\
\hline
\end{tabular}

Fuente: Elaborado a partir de datos recolectados por los investigadores.

Asimismo, al comparar las relaciones generadas por el software con la opinión de expertos en la materia surgen algunas discrepancias, esto se debe a que los grupos generados por el SPSS se basan en la información alimentada de la base de datos generada y esto no garantiza que las relaciones obtenidas sean $100 \%$ exactas. Por último, a pesar de encontrar errores en un par de estas relaciones cabe destacar que si es posible ver un patrón en los resultados obtenidos.

\subsection{Procedimiento}

El procedimiento que se siguió para obtener los resultados fue el siguiente: 1) Realizar prueba piloto aplicando la rúbrica a una parte de la muestra; 2) Obtener los datos de la evaluación, aplicando la rúbrica al resto de la muestra establecida; 3) Interpretar los resultados de la evaluación, a partir de gráficos elaborados; 4) Documentar el mecanismo de evaluación validado, considerando los lineamientos de normas internacionales.

\section{RESULTADOS Y DISCUSIÓN}

Al aplicar la rúbrica a los primeros trabajos se detectaron algunos errores en la redacción de algunos puntos que hacían que la evaluación fuera un poco confusa, por lo que se replantearon, además de esto se decidió disminuir la escala de Likert de cinco a cuatro calificaciones. Posteriormente, se aplicó la versión definitiva de la rúbrica hasta completar la muestra de 97, que se categorizó en cuatro rubros: proyectos elaborados en materias del bloque de logística, así como los realizados en el curso de análisis de sistemas con apoyo de la Metodología de Análisis de una Organización (MAO) propuesta por Arellano-González y Carballo-Mendívil (2013), en sU versión 1, 2 y 3 (MAO ver1, MAO ver2 y MAO ver3).

Los resultados obtenidos se resumen en la Figura 1, donde se muestra el nivel de cumplimiento que se calculó utilizando los datos obtenidos de la evaluación, que fueron ponderados según su importancia en las competencias analizadas.

En esta figura 1 es posible observar el impacto que tiene la versión de la metodología MAO en el nivel de cumplimiento de las distintas muestras, siento la última versión en conjunto con el enfoque que se le ha dado a la materia este último semestre las que han obtenido el mayor nivel de cumplimiento de las cuatro muestras. 
Blanca Carballo Mendívil, Alejandro Arellano González, José Mario Salomón González Evaluación de las habilidades requeridas en el análisis de un sistema organizacional

Figura 1 Nivel de CUMPLIMIENTO POR MUESTRA DE LOS PRODUCTOS ELABORADOS POR LOS ALUMNOS.

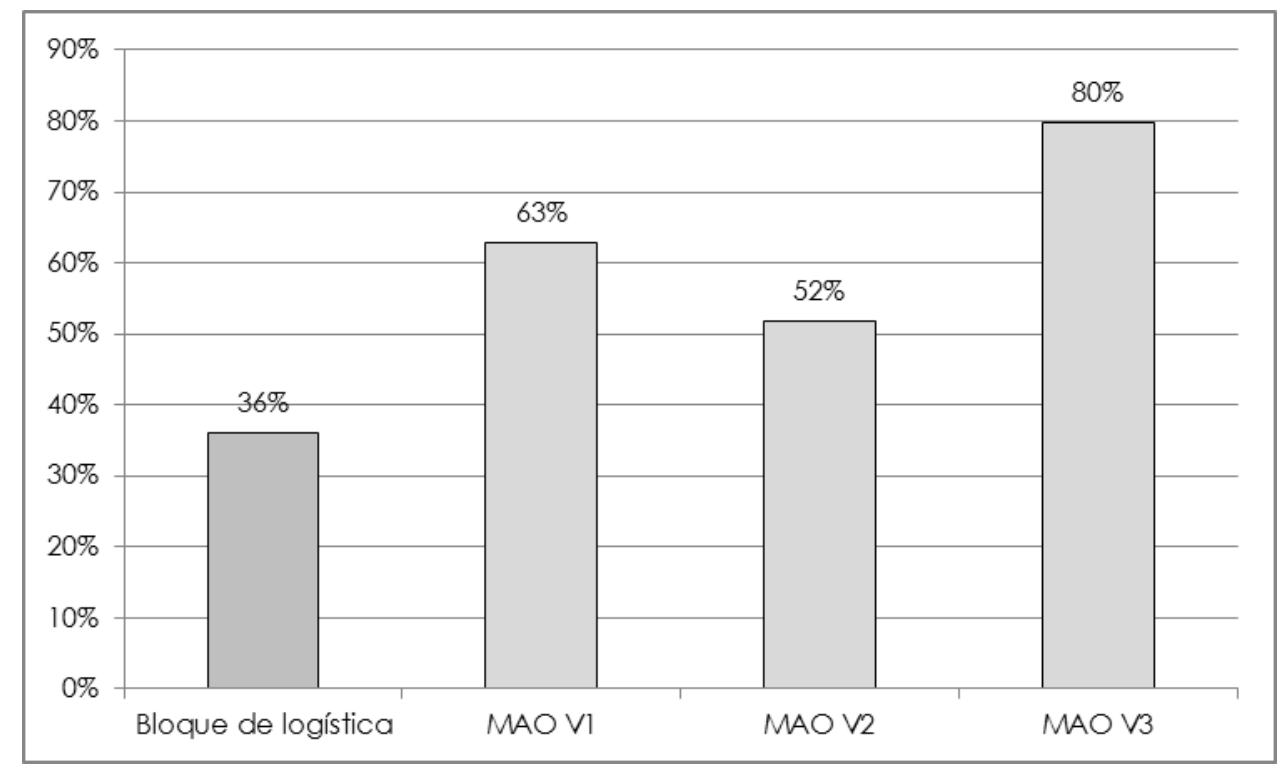

Resultados más detallados se presentan en la Figura 2 donde se muestran los valores máximos, mínimos y medias del nivel de cumplimiento obtenido en la evaluación para las cuatro muestras evaluadas, en la cual se observa el mismo comportamiento en las calificaciones máximas y mínimas obtenidas, aunque las muestras que presentan un rango menor entre estos dos valores son el bloque de logística y la versión 3 de la $M A O$.

FIGURA 2 PUNTAJES PROMEDIO MÁXIMOS, MEDIOS Y MÍNIMOS OBTENIDOS POR MUESTRA.

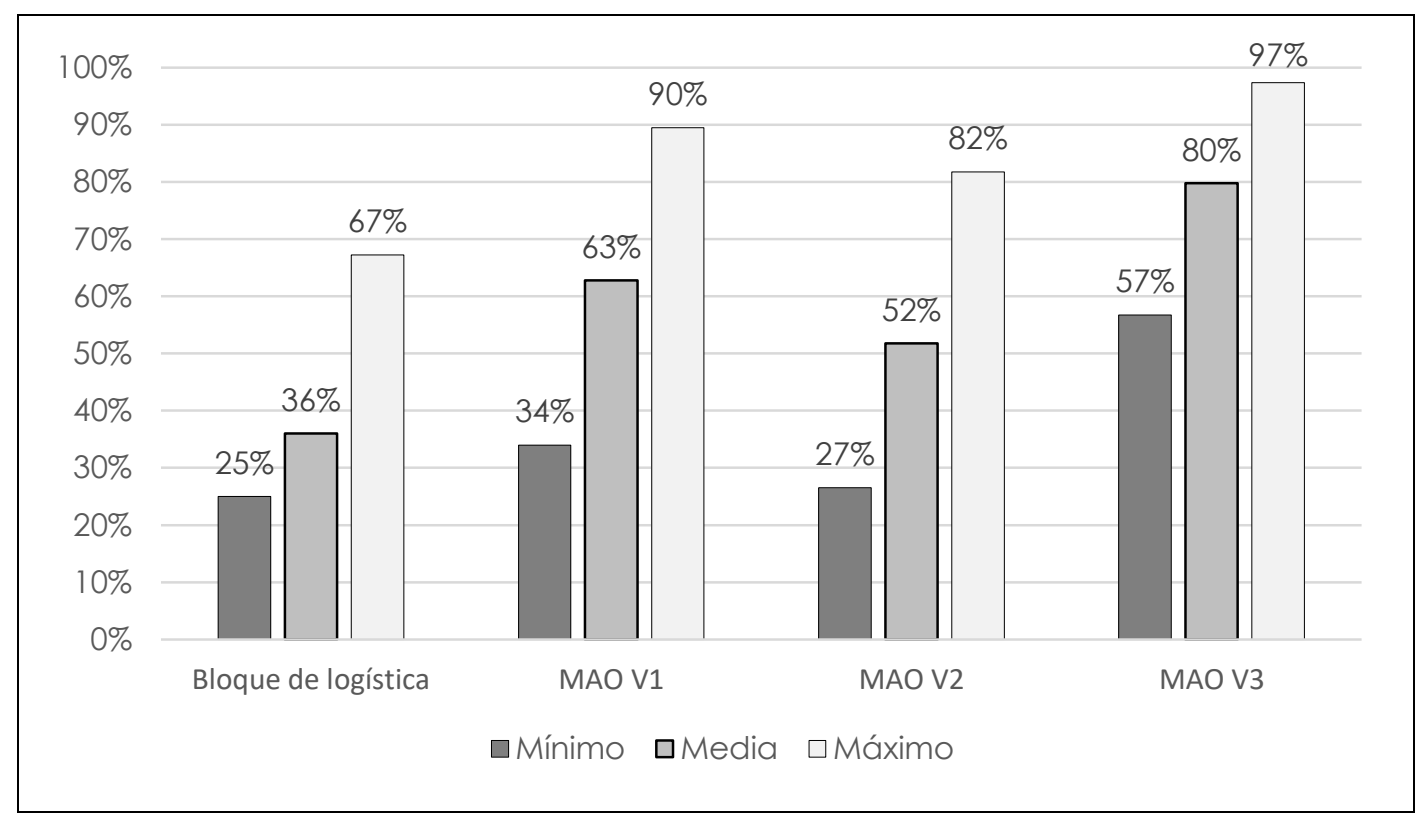

Fuente: Elaborado a partir de datos recolectados por los investigadores.

Como se puede observar en las gráficas anteriores, los resultados obtenidos en la mayoría de los ítems es mayor en los trabajos que se apoyaron en la metodología MAO para la realización del análisis, a su vez, dentro de los productos que fueron desarrollados utilizando esta metodología se puede observar que los elaborados con la versión 3 son los que tienen mejores resultados en todos los ítems evaluados. 
Asimismo, se observó los ítems que representan la fase descriptiva de la organización presentan una menor calificación que aquellos donde se realiza el análisis.

Además, a continuación se muestra gráficos de forma radial donde se identifican los resultados obtenidos en cada uno de los elementos evaluados por muestra: bloque logístico, MAO V1, MAO V2 y MAO V3.

En la Figura 3 se presentan los resultados de la evaluación realizada a los proyectos desarrollados en el bloque de logística, que son cursados por alumnos que ya deberían haber adquirido las competencias de analista. Como se observa, las calificaciones obtenidas en esta muestra son bajas, siendo menores a 2 puntos, donde 4 es el máximo puntaje posible, esto significa que todos los elementos son desarrollados por debajo de las especificaciones.

Figura 3 Nivel de CUMPLIMIENTO PROMEdio POR ÍtEM EN LOS PROYeCTOS DEL blOQUE DE LOGÍSTICA.

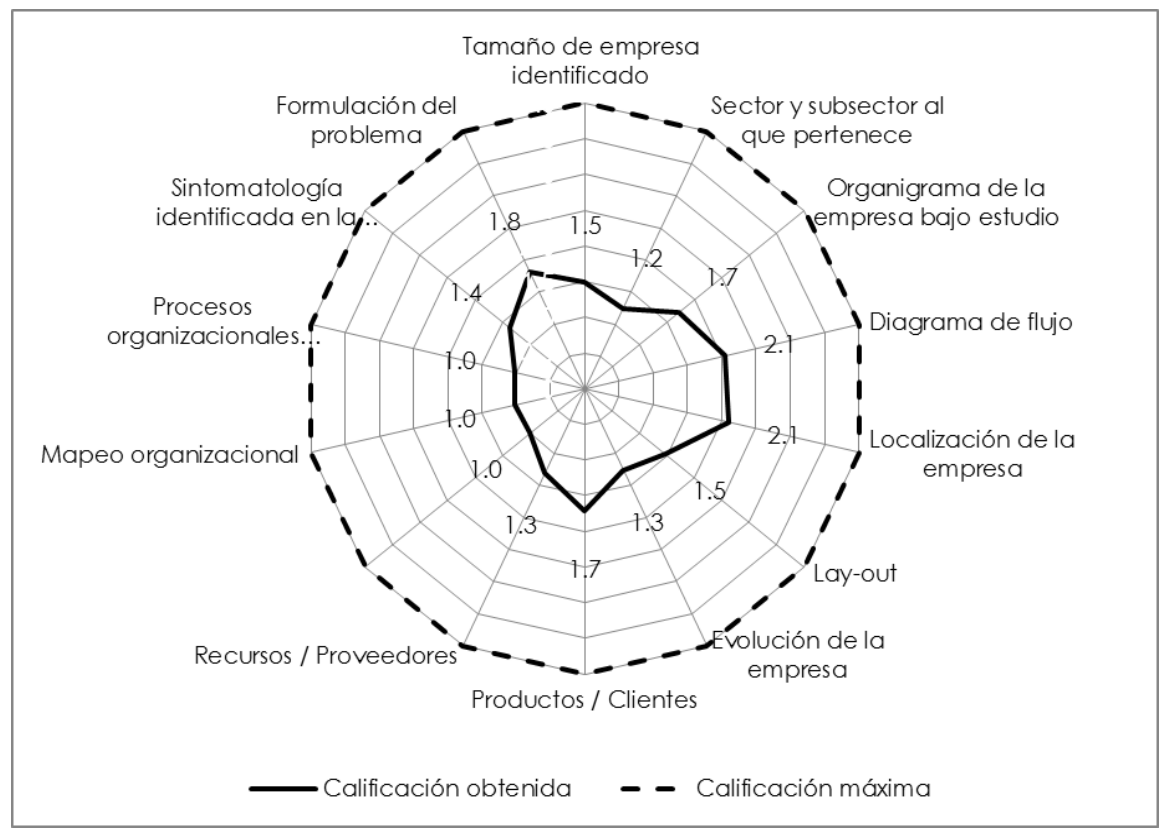

Fuente: Elaborado a partir de datos recolectados por los investigadores.

Para apreciar mejor los promedios de todos los ítems en esta muestra se presenta la Tabla 4, donde se ordenan por promedio de menor a mayor promedio. Es importante recordar que en el bloque de logística los alumnos ya adquirieron las habilidades de analista, pero como se observa en los resultados mostrados en esta Tabla 1, los puntajes promedio parecen decir lo contrario.

En esta Tabla 4 se observa que todos los elementos evaluados están por debajo de las especificaciones. Por mencionar algunos, en el ítem localización de la empresa se encontró que la mayoría de los trabajos evaluados solo tenían una breve descripción de la dirección y foto de la empresa sin mucha explicación ni con el contenido que se establece como requisito; el diagrama de flujo no fue desarrollado o los errores eran tales que se tenía que volver a hacer (sólo el 9\% cumplió con todo lo que se pide que es una breve introducción del diagrama, el diagrama desarrollado correctamente y la descripción de los pasos del diagrama); y la formulación del problema, que es lo más importante para determinar la habilidad de analista en el alumno, y aunque todos los proyectos indicaban cual era el problema, ninguno contaba con datos que permitieran identificar de manera objetiva las brechas que existían entre el estado real y lo deseado. 
TABla 4 Agrupación de Ítems.

\begin{tabular}{ll}
\hline Elementos evaluados en proyectos de logística & Puntuación promedio \\
\hline Contexto Organizacional: PESTE & 1.00 \\
Mapeo organizacional & 1.02 \\
Procesos organizacionales idealizados & 1.02 \\
Sector y subsector al que pertenece & 1.23 \\
Evolución de la empresa & 1.26 \\
Recursos / Proveedores & 1.31 \\
Sintomatología identificada en la organización & 1.36 \\
Lay-out & 1.47 \\
Tamaño de empresa identificado & 1.50 \\
Organigrama de la empresa bajo estudio & 1.71 \\
Productos / Clientes & 1.71 \\
Formulación del problema & 1.81 \\
Diagrama de flujo & 2.05 \\
Localización de la empresa & 2.10 \\
\hline
\end{tabular}

Fuente: Elaborado a partir de datos recolectados por los investigadores.

Un punto importante que se debe de mencionar es que en el bloque de logística no se les brinda a los alumnos una metodología de apoyo, como la MAO, y también que estos cursos de logística más allá de un análisis se enfoca más en planear sistemas logísticos, en la demanda de inventarios y en los sistemas de almacenamiento. Sin embargo, todo alumno que cursa estas materias fue habilitado previamente en el análisis con el uso de esta metodología, y que además todos los trabajos nacen de un problema el cual se debe determinar en la organización y es en esto en lo que están fallando los alumnos.

Por otro lado, en la Figura 4 se presentan los resultados de otra muestra que pertenece a los proyectos desarrollados en la materia de análisis de sistemas en los semestres Agosto - Diciembre del 2010 y Enero - Mayo del 2011, específicamente con el uso de la primera versión de la metodología MAO.

Figura 4 Nivel de CUMPLIMIENTO PROMEdio POR Ítem EN los PROYeCtOS Elaborados CON la MAO V1.

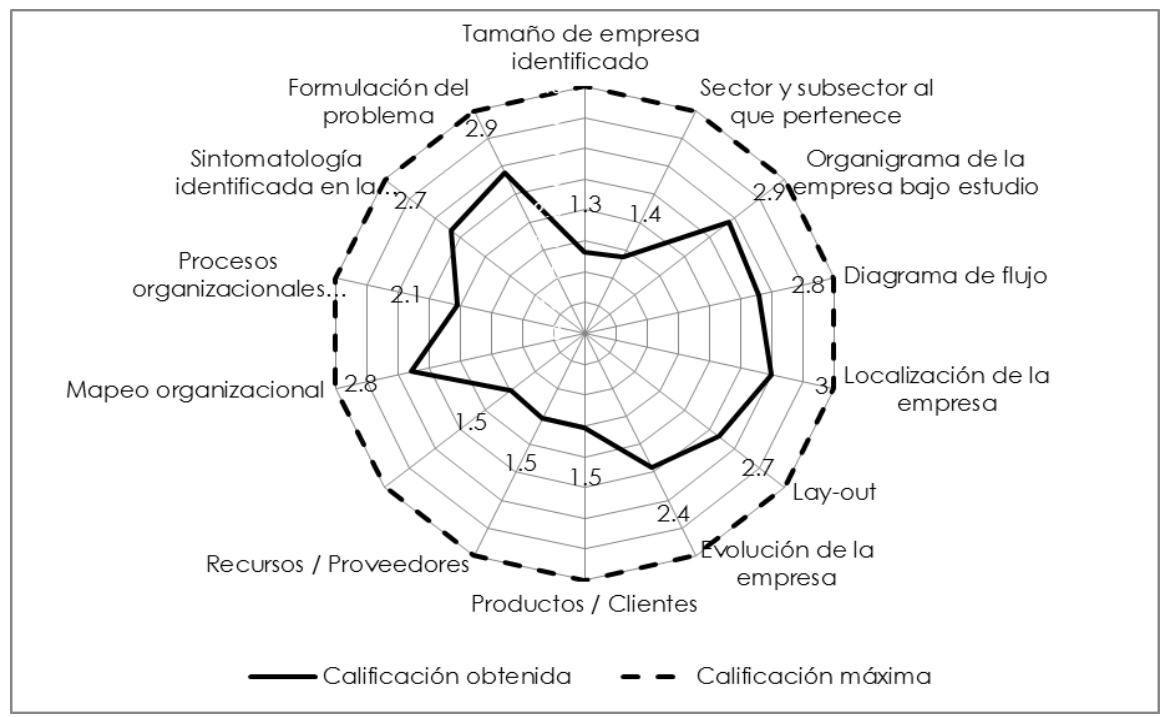

Fuente: Elaborado a partir de datos recolectados por los investigadores. 
En esta gráfica de la Figura 4 se puede observar que los puntajes aún siguen muy lejos de la calificación máxima de 4 puntos, es decir, algunos elementos no son desarrollados o se consideran por debajo de las especificaciones.

Para apreciar mejor los promedios de todos los ítems en esta muestra se elaboró la Tabla 5, donde están ordenados de menor a mayor la puntuación promedio. Se observa que existe un ítem con promedio regular (Localización de la empresa) y otros más cercanos al 2 (Formulación del problema y organigrama de la empresa bajo estudio) lo que quiere decir que se obtuvieron buenas calificaciones, aunque también hay 5 (tamaño de empresa identificado, sector y subsector al que pertenece, PESTE, productos/clientes, y recursos/proveedores) ítems que se tienen que seguir mejorando ya que están por debajo de lo regular.

tabla 5 Resultados de la eValuación de los proyectos elaborados con la MaO V1.

\begin{tabular}{ll}
\hline Elementos evaluados en proyectos elaborados con la MAO V1 & Puntuación promedio \\
\hline Tamaño de empresa identificado & 1.31 \\
Sector y subsector al que pertenece & 1.36 \\
Contexto Organizacional: PESTE & 1.47 \\
Productos / Clientes & 1.52 \\
Recursos / Proveedores & 1.52 \\
Procesos organizacionales idealizados & 2.05 \\
Evolución de la empresa & 2.42 \\
Lay-out & 2.68 \\
Sintomatología identificada en la organización & 2.68 \\
Mapeo organizacional & 2.78 \\
Diagrama de flujo & 2.78 \\
Organigrama de la empresa bajo estudio & 2.89 \\
Formulación del problema & 2.89 \\
Localización de la empresa & 3.00 \\
\hline
\end{tabular}

Fuente: Elaborado a partir de datos recolectados por los investigadores.

En esta segunda muestra MAO V1, los promedios son más aceptables que la muestra anterior. Por ejemplo, para la localización de la empresa se presenta un mapa macro y micro de la empresa con la dirección, además de una breve explicación de la localización, lo cual se considera regular; asimismo, en la formulación del problema se mostraba una lista de síntomas identificados en cada proceso, a partir de los cuales se planteaban los problemas, que es lo más importante del análisis.

Asimismo, en la Figura 5 se presentan los resultados de la tercera muestra correspondiente a los proyectos elaborados con uso de la MAO versión 2, en la materia de análisis de sistema durante los periodos Agosto - Diciembre del 2011 y Enero - Mayo del 2012, observándose una mejoría con respecto a la muestra anterior de manera general, aunque la formulación del problema presentó muy bajo puntaje, lo cual indica que en esta muestra no se encontraron evidencias suficientes que indiquen que el alumno ha desarrollado la competencia de análisis. 


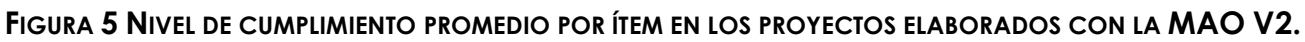

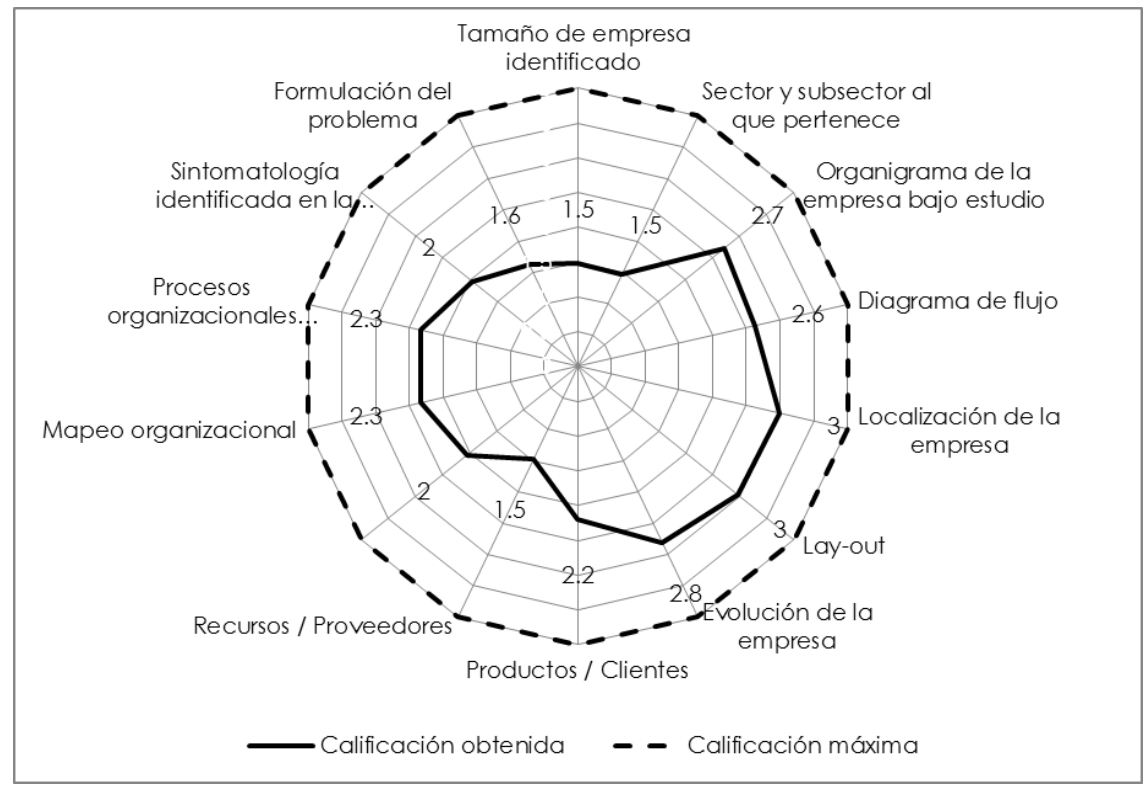

En la Tabla 6 se presentan ordenados los promedios obtenidos por ítem, de menor a mayor promedio para poder identificar con mayor facilidad cuáles son aquellos elementos que el alumno desarrolló en esta muestra. En este caso un sólo ítem que es regular el cual es localización de la empresa, y 3 más que casi son regulares, se tienen que tomar en cuenta los últimos cuatro ítems ya que salieron muy por debajo de las especificaciones y deben ser revisados.

TABLA 6 Resultados de la eVAluación de los proyectos elaborados Con la MAO V2.

\begin{tabular}{ll}
\hline Elementos evaluados en proyectos elaborados con la MAO V2 & Puntuación promedio \\
\hline Tamaño de empresa identificado & 1.47 \\
Recursos / Proveedores & 1.47 \\
Sector y subsector al que pertenece & 1.47 \\
Formulación del problema & 1.61 \\
Sintomatología identificada en la organización & 1.95 \\
Contexto Organizacional: PESTE & 2.04 \\
Productos / Clientes & 2.19 \\
Mapeo organizacional & 2.33 \\
Procesos organizacionales idealizados & 2.33 \\
Diagrama de flujo & 2.61 \\
Organigrama de la empresa bajo estudio & 2.71 \\
Evolución de la empresa & 2.80 \\
Lay-out & 2.95 \\
Localización de la empresa & 3.00 \\
\hline
\end{tabular}

Fuente: Elaborado a partir de datos recolectados por los investigadores.

Por último, en la Figura 6 se presentan los resultados de la evaluación de la muestra mejor evaluada, que corresponden a los proyectos elaborados en la materia de análisis de sistema con apoyo en la metodología MAO versión 3, utilizada en Enero - 
Mayo del 2013. Se puede notar que la mayoría de los promedios están por arriba de 3, es decir, son mejores que regular.

Figura 6 Nivel de CUMPLIMIENTO PROMEDIO POR ÍtEM EN lOS PROYECTOS ELABORAdOS CON LA MAO V3.

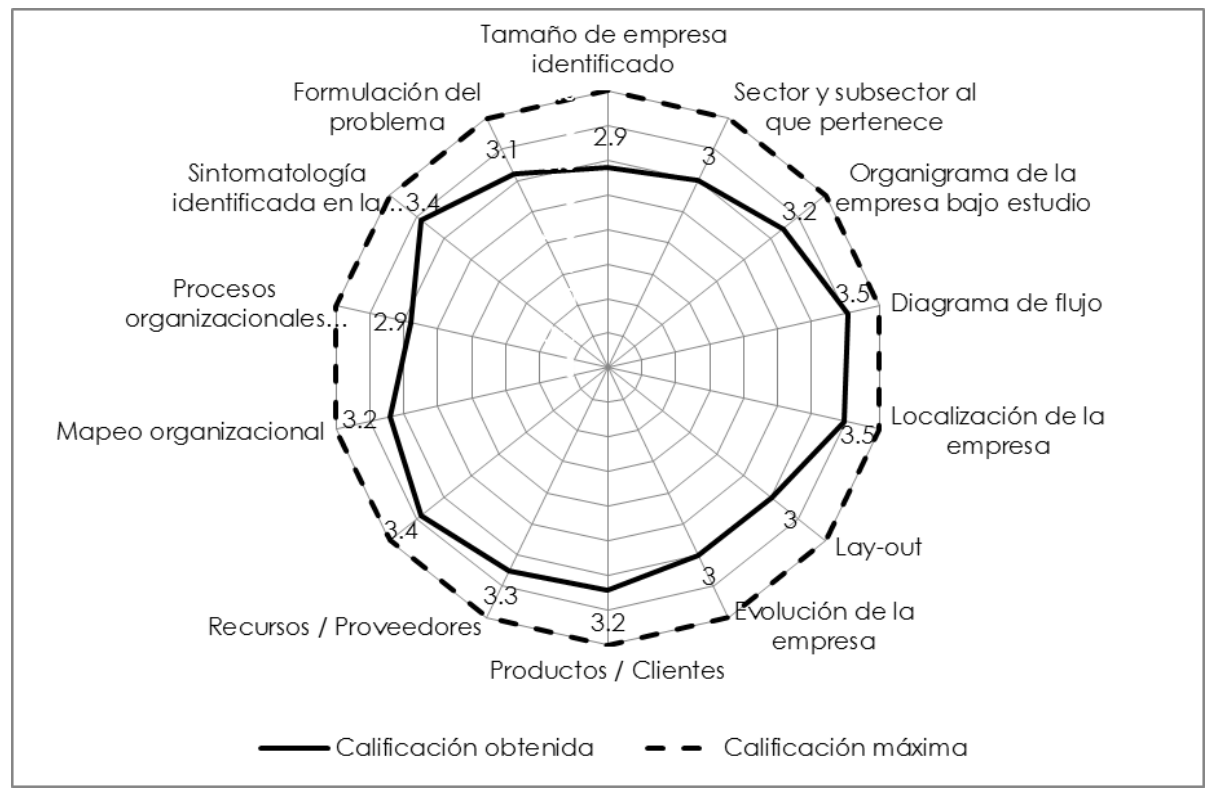

Fuente: Elaborado a partir de datos recolectados por los investigadores.

Para apreciar mejor los resultados de esta muestra, en la Tabla 7 se presentan ordenados de menor a mayor los promedios de todos los ítems, donde se puede apreciar que casi todos sobrepasan un puntaje de 3, es decir, están en un rango de regular a bueno, a excepción de dos (tamaño de la empresa identificado, y procesos organizacionales idealizados).

TABla 7 Resultados de la eVAlUación de los proyectos elaborados con la MAO V3.

\begin{tabular}{ll}
\hline Elementos evaluados en proyectos elaborados con la MAO V3 & Puntuación promedio \\
\hline Tamaño de empresa identificado & 2.89 \\
Procesos organizacionales idealizados & 2.89 \\
Sector y subsector al que pertenece & 3.00 \\
Lay-out & 3.00 \\
Evolución de la empresa & 3.00 \\
Formulación del problema & 3.10 \\
Organigrama de la empresa bajo estudio & 3.21 \\
Productos / Clientes & 3.21 \\
Mapeo organizacional & 3.21 \\
Recursos / Proveedores & 3.26 \\
Contexto Organizacional: PESTE & 3.42 \\
Sintomatología identificada en la organización & 3.42 \\
Localización de la empresa & 3.47 \\
Diagrama de flujo & 3.52 \\
\hline
\end{tabular}

Fuente: Elaborado a partir de datos recolectados por los investigadores. 
En los promedios de esta muestra se obtuvo el puntaje mayor de 3.52 en el ítem correspondiente al diagrama de flujo, es decir, los trabajos están por arriba de lo regular, ya que cuentan con el diagrama una breve introducción y una explicación al final del diagrama, es claro y se describen las actividades realizadas; asimismo el ítem de localización de la empresa, igualmente está por arriba de lo regular, ya que se presenta un mapa con la macro y micro localización así como una breve descripción con la dirección de la empresa, estado, ciudad, colonia y foto de la fachada. Pero lo más importante es que el ítem de sintomatología identificada en la organización, también obtuvo un puntaje bueno de 3.42, es decir, la mayoría de los proyectos desarrollados por los alumnos presentan dato duro sobre la situación actual no deseada de la empresa, se apoya de indicadores críticos y se describen las consecuencias negativas que tiene la organización.

Como se observa, aunque los resultados de esta muestra son mejores que las anteriores, al analizar los resultados de manera integral, se observa que los elementos que son desarrollados en mayor y menor medida en las cuatro muestras son los que se listan en la Tabla 8, es decir, los ítems mejor evaluados en todos los trabajos.

TABLA 8 Elementos EVALUAdOS CON MAYOR Y MENOR PUNTAJE OBTENIDO.

\begin{tabular}{ll}
\hline Elementos con mayor puntaje obtenido & Elementos con menor puntaje obtenido \\
\hline Localización de la empresa & Tamaño de empresa identificado \\
Diagrama de flujo & Sector y subsector al que pertenece \\
Formulación del problema & Contexto Organizacional: PESTE \\
& Procesos organizacionales idealizados \\
\hline
\end{tabular}

Fuente: Establecido a partir de datos recolectados por los investigadores.

Es evidente que los resultados obtenidos en la evaluación de la mayoría de los ítems obtuvo un mayor puntaje en los trabajos que se apoyaron en alguna metodología para la realización del análisis. Además, queda claro que la versión 3 de la metodología MAO que se utiliza en la materia de análisis de sistemas, es la que permite generar producto de mejor calidad. Además, comparando los resultados de las cuatro muestras es posible notar que los ítems que representan la fase descriptiva de la organización presentan una menor calificación que aquellos donde se realiza el análisis, lo que podría significar que los alumnos no consideran importante describir la empresa que estudian, sino sólo cumplir con el objetivo del proyecto.

Por último, es de relevancia resaltar la importancia que tiene la evaluación continua no sólo del desempeño mostrado por los alumnos en las aulas, sino de las evidencias de producto que generan en todos sus curso, las cuales dan evidencia del desarrollo de las competencias que se tienen establecidas en los programas educativos. Por eso, en la Figura 7 se muestra el diagrama de flujo del procedimiento que se propone debe seguirse para replicar la evaluación aplicada a los productos generados por los alumnos para determinar el grado en que han desarrollado las habilidades requeridas en el análisis de un sistema organizacional, una competencia básica para un futuro egresado.

En la Figura 7 se muestran las actividades clave para llevar a cabo una evaluación de habilidades, el cual empieza al definir el alcance del proyecto con el encargado que es el coordinador de la academia y evaluadores, seguido de solicitar los productos a evaluar, revisar la metodología que se utilizó (MAO V3), si se tiene que modificar la 
metodología respecto a la que se está utilizando, aplicar el instrumento, procesar la información de la evaluación, al final elaborar informe de resultados.

FIgURA 7 MECANISMO PARA LA EVALUACIÓN DE PROYECTOS DESARROLLADOS EN CURSOS CURRICULARES bASADOS EN UNA METODOLOGÍA.

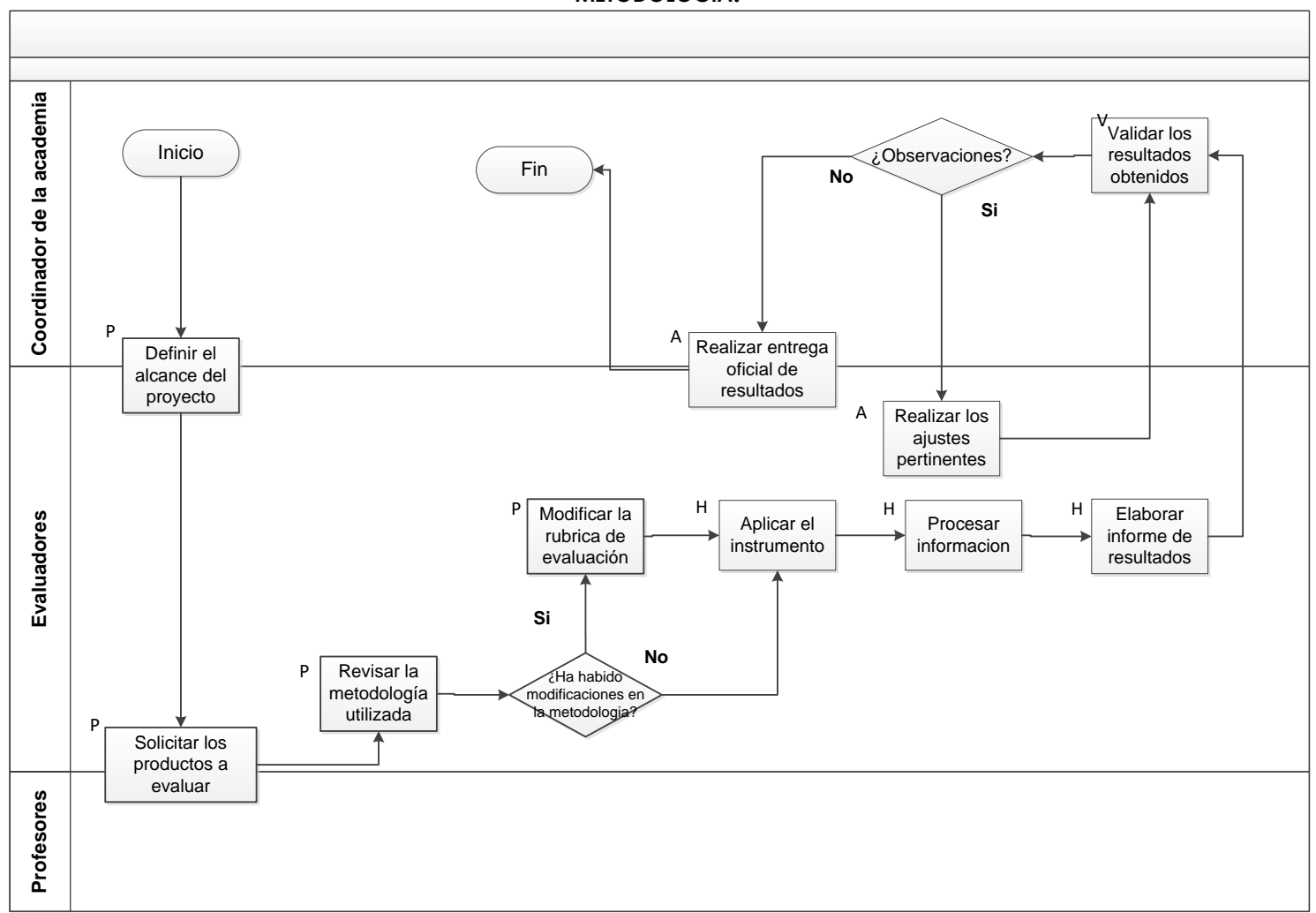

Fuente: Elaborado por los investigadores

Como se observa en la figura 7, el diagrama original está codificado utilizando cuatro diferentes colores (por restricciones del formato de la revista no se pueden mostrar así), mismos que indican un ciclo de mejora continua: Planear-Hacer-Verificar-Actuar (PHVA); a lado izquierdo de cada actividad en el diagrama se indica la letra inicial correspondiente a cada una de estas fases. Este procedimiento permitirá que el estudio realizado pueda ser replicado las veces que se desee y que evolucione de acuerdo a las necesidades que surjan.

\section{CONCLUSIONES Y RECOMENDACIONES}

Después de haber analizado los resultados obtenidos se puede concluir que se cumplió el objetivo ya que se logó diseñar un mecanismo de evaluación respecto a las habilidades requeridas en el análisis de un sistema organizacional para determinar el nivel de competencia adquirido por los alumnos. La rúbrica de evaluación y los materiales diseñados pueden ser aplicados para la evaluación de las habilidades de análisis y el método documentado puede seguirse para diseñar nuevos instrumentos de evaluación, y así orientar los programas educativos hacia la mejora continua.

Es importante que las universidades, en este caso ITSON, compruebe que los profesionistas que está formando estén adquiriendo las competencias que establece el programa educativo, es por eso que se considera necesario que se dé seguimiento a esta situación y se siga aplicando los instrumentos (o nuevas versiones) aquí presentados. 
El diseñar un instrumento de evaluación no es una tarea simple, ya que se es necesario considerar la mayor cantidad de escenarios que se puedan presentar dentro de las delimitaciones de la evaluación, pero es necesario para que todos los productos bajo estudio puedan ser evaluados bajos los mismos criterios, sin importar quién sea el evaluador.

Gracias a los análisis estadísticos ha sido posible comprobar la confiabilidad del instrumento generado, por lo que se considera que dicho instrumento es apto para seguirse aplicando siempre y cuando las condiciones de evaluación no cambien (requerimientos establecidos en la MAO así como la estructura y enfoque del curso de Análisis de Sistemas).

Además de la evaluación de los productos, la rúbrica puede ser utilizada por los alumnos para realizar una auto-evaluación, de esta manera identificar con que elementos cumple o que elementos es necesario seguir desarrollando, esto ayudará a que identifiquen sus fallas antes de que su profesor se las haga indique.

Debido a que el nivel de cumplimiento de las habilidades para el análisis de un sistema organizacional aparentemente se pierde una vez aprobado el curso, se recomienda que se dé un seguimiento a las competencias adquiridas en cursos anteriores, específicamente en el caso de análisis, se recomienda que en las materias posteriores a este bloque se establezca como requisito usar la metodología MAO como apoyo para elaborar los antecedentes de los proyectos que se desarrollen.

Es importante que el resto de los bloques de la carrera, así como el resto de los programas educativos se preocupen por evaluar el desarrollo de las habilidades y conocimientos que los alumnos están adquiriendo en las aulas, para ser capaces de detectar áreas de oportunidad, estandarizar la calidad de los proyectos desarrollados y poder así formar mejores profesionistas acordes con los perfiles de egreso establecidos.

\section{BIBLIOGRAFÍA}

Alarcón-Ortiz, D., \& Freire-Cruz, T. (2012). Mejora del desempeño en el trabajo en equipo como función de las dimensiones culturales: solidaridad y sociabilidad en el comportamiento organizacional. Negotium, 7(21), 18-29.

Alfaro-Tanco, J. A., Rodríguez-Chacón, V., \& Amorrortu-Gervasio, I. (2014). Desarrollo de competencias y habilidades a través de proyectos basados en empresas reales: Análisis en asignaturas de Dirección de Operaciones. Educade - Revista de Educación en Contabilidad, Finanzas y Administración de Empresas(5), pendiente.

Alvarez-Cáceres, R. (1995). Estadística multivariante y no paramétrica con SPSS. Aplicación a las ciencias de la salud. Madrid: Diaz de Santos.

Apellániz, J. M., \& Amayra, I. (2008). La forma del dibujo figurativo paleolítico a través de la experimentación: Una aproximación desde la prehistoria y la psicología cognitiva. Bilbao: Universidad Deusto.

Ardila-Soto, V. M., \& Gómez-Chiñas, C. (2005). Trabajo en equipo: el caso colombiano. Análisis Económico, XX(43), 147-165.

Arellano González, A., \& Carballo Mendívil, B. (2013). Metodología de análisis de una organización (vista como un sistema). Ciudad Obregón: No publicada.

Arellano-González, A., \& Carballo-Mendívil, B. (2011). Desarrollo de proyectos como estrategia para el logro de las competencias profesionales del Ingeniero Industrial y de Sistemas. En R. I. Pizá, Y. Moreno, \& G. Marisela, Desempeño 
profesional para el seguimiento de competencias (págs. 45-55). Ciudad Obregón: Instituto Tecnológico de Sonora.

Arellano-González, A., Ríos-Vázquez, N. J., Carballo-Mendívil, B., Montero-Quintero, J. A., \& Tapia-Mimila, D. A. (2013). Solución tecnológica para mejorar el desempeño académico y disminuir el rezago educativo. Ciudad Obregón: Instituto Tecnológico de Sonora.

Bernal-Torres, C. A. (2006). Metodología de la investigación: Para administración, economía, humanidades y ciencias sociales (2da ed.). México: Pearson Educación.

Capote, S., \& Sosa, Á. (Diciembre de 2006). Evaluación: Rubrica y listas de control. Obtenido de Colegio La Asunción: http://www.josefinastrinitarias.org/laasuncionc/PJ/aplicaciones/adj/example/files/Evaluacin.pdf

Galeana de la O., L. (2006). Aprendizaje basado en proyectos. Revista CEUPROMED, 117.

García-Almiñana, D., \& Amante García, B. (2006). Algunas experiencias de aplicación del aprendizaje cooperativo y del aprendizaje basado en proyectos. I Jornadas de Innovación Educativa (págs. 1-7). Zamora: Escuela Politécnica Superior de Zamora.

García-Fernández, F., \& Cordero-Borjas, A. E. (2007). Equipos de trabajo: forma organizativa de la economía basada en el conocimiento. Economía y Sociedad, XII(20), 17-33.

Guerra-López, I., \& Rodriguez-Villanueva, G. (2005). Educational Planning and Social Responsibility: Eleven Years of Mega Planning at the Sonora Institute of Technology (ITSON). Performance Improvement Quarterly, 18 (3), 56-64.

Hernández-Alvarez, J. L., Velázquez-Buendía, R., Alonso-Curiel, D., Castejón-Oliva, F. J., Garoz-Puerta, I., López-Crespo, C., y otros. (2004). La evaluación en educación física: investigación y práctica en el ámbito escolar. España: Editorial GRAO.

Instituto Tecnológico de Sonora. (2009). Modelo curricular ITSON. Obtenido de Inovación Curricular: http://www.itson.mx/empleados/servicios/innovacion/Documents/MODELO_CU RRICULAR_ITSON_2009.pdf

Kolmos, A. (2004). Estrategias para desarrollar currículos basados en la formulación de problemas y organizados en base a proyectos. Revistas DIALNET, 33, 77-96.

Labra-Gayo, J. E., Fernández Lanvin, D., Calvo Salvador, J., \& Cernuda del Río, A. (2006). Utilización de herramientas colaborativas de desarrollo de software libre en un modelo de aprendizaje basado en proyectos. XII Jornadas de Enseñanza Universitaria de Informática (págs. 1-8). Bilbao: Universidad Politécnica de Valencia.

López-Hernández, A., \& Abelló-Planas, L. (2007). El desarrollo de competencias docentes en la formación del profesorado. España: Ministerio de educación.

Lucero, I., \& Meza, S. (2002). Validacion de instrumentos para medir conocimientos. Ciencia \& Técnica. Comunicaciones científicas y tecnológicas, III(4), 1-10.

Maldonado-Pérez, M. (2008). Aprendizaje basado en proyectos colaborativos. Una experiencia en educacion superior. Laurus, 14 (28), 1-24.

Méndez-Fregozo, E. (2005). La Acción Urgente: Un Modelo Innovador de estructura organizacional para la Universidad Pública del Siglo Veintiuno. Guadalajara: Universidad de Guadalajara.

Molina-Morales, F. X., Martínez-Fernández, M. T., Ares-Vázquez, M. Á., \& Emil-Hoffmann, V. (2008). La estructura y naturaleza del capital social en las aglomeraciones territoriales de empresas. Una aplicación al sector cerámico español. España: Fundación BBVA.

Pech-Pech, A., Castillo-León, T., \& Echeverría-Echeverría, R. (2005). Diagnóstico de Competencias Profesionales para el Trabajo en Equipos Interdisciplinarios en una Unidad de Salud. Psicología Iberoamericana, 13(1), 12-19. 
Blanca Carballo Mendívil, Alejandro Arellano González, José Mario Salomón González Evaluación de las habilidades requeridas en el análisis de un sistema organizacional

Tenbrink, T. D. (2006). Evaluacion. Guia práctica para profesores. Madrid: Narcea.

Valero-García, M. (2005). Las dificultades que tienes cuando haces PBL. La Educación Superior hacia la Convergencia Europea: Modelos basados en el aprendizaje (págs. 1-8). Mondragón: Universidad de Mondragón. 